\title{
EFEITO DA ADIÇÃO DE CINZA DA CASCA DE ARROZ EM MISTURAS CIMENTO-CASCA DE ARROZ ${ }^{1}$
}

\author{
LORIS L. ZUCCO², ANTONIO L. BERALDO ${ }^{3}$
}

RESUMO: A casca de arroz e sua cinza são abundantes e renováveis, podendo ser empregadas na obtenção de materiais de construção alternativos. O aumento do consumo desses resíduos poderia ajudar a minimizar os problemas ambientais provenientes da sua eliminação inadequada. Este trabalho teve o objetivo de avaliar a utilização de cinzas como carga mineral (filler). Todavia, a casca de arroz interferiu quimicamente no comportamento das misturas à base de cimento. Assim, diferentes misturas cimento-casca de arroz, com e sem adição de cinzas, foram avaliadas, a fim de destacar a influência de seus componentes (casca; cinza) que, de outra forma, poderiam ser excluídas ou subestimadas. Amostras cilíndricas (teste de compressão simples e de tração por compressão diametral) e amostras extraídas das placas prensadas (teste de flexão e compressão paralela à superfície) foram usadas para avaliar o comportamento das misturas e dos componentes casca e cinza. Os resultados dos ensaios mecânicos mostraram, em geral, que não houve diferença estatística entre as misturas, as quais estão associadas ao efeito químico supressivo da cinza da casca de arroz. A mistura da casca de arroz de $10 \mathrm{~mm}$ com o acréscimo de $35 \%$ das cinzas destacase por permitir o mais elevado consumo de casca e cinzas, reduzir $25 \%$ no consumo de cimento e permitir o confinamento (sem emissões para a atmosfera) de cerca de 1,9 tonelada de $\mathrm{CO}_{2}$ por tonelada de cimento consumido, contribuindo, assim, para a redução da emissão de $\mathrm{CO}_{2}$, o que pode incentivar construções rurais sob o ponto de vista ecológico.

PALAVRAS-CHAVE: casca de arroz, cinza, compósito.

\section{EFFECT OF THE ADDITION OF RICE HUSK ASH IN CEMENT-RICE HUSK MIXTURES}

\begin{abstract}
The rice husk and its ash are abundant and renewable and can be used to obtain alternative building materials. An increase in the consumption of such waste could help minimize the environmental problems from their improper disposal. This study aimed to evaluate the use of ashes as a cargo mineral (filler). However, the rice husk chemically interferes in the conduct of the based cement mixtures. Thus, different mixes cement-rice husk with and without the addition of ash were evaluated in order to highlight the influence of its components (husk; ash), which could otherwise be excluded or be underestimated. Cylindrical samples (test of simple compression and traction by diametrical compression) and samples extracted from manufactured pressed board (test of bending and parallel compression to the surface), were used to evaluate the behavior of different mixtures of components (rice hush; RHA - rice husk ahs). The results of the mechanical tests showed, in general, there is not a statistical difference between the mixtures, which are associated with the chemical suppressive effect of the rice husk ash. The mixture of rice husk of $10 \mathrm{~mm}$, with an addition of $35 \%$ of the rice husk ash, is notable for allowing the highest consumption of rice husk and rice husk ash, to reduce $25 \%$ the consumption of cement and to allow the storage (without emissions to the atmosphere), around 1.9 ton of $\mathrm{CO}_{2}$ per ton of cement consumed, thus contributing to the reduction of $\mathrm{CO}_{2}$ emissions, which can stimulate rural constructions under an ecological point of view.
\end{abstract}

KEYWORDS: composite, rice husk, rice husk ash (RHA).

\footnotetext{
${ }^{1}$ Extraído da tese de Doutorado do primeiro autor.

${ }^{2}$ Licenciado em Ciências Agrícolas, Engo Agrônomo, Professor, UFRRJ/CTUR, Seropédica - RJ, Fone/Fax: (0XX21) 2682.1004, 1lzucco@ufrrj.br

${ }^{3}$ Eng $^{\mathrm{O}}$ Agrícola, Prof. Associado, FEAGRI/UNICAMP, Caixa Postal 6011, Campinas - SP, beraldo@agr.unicamp.br

Recebido pelo Conselho Editorial em: 3-7-2007
}

Aprovado pelo Conselho Editorial em: 25-5-2008 


\section{INTRODUÇÃO}

O aproveitamento de resíduos agrícolas tem-se tornado uma necessidade em função do alto custo ambiental derivado do seu inadequado descarte na natureza. Nesse cenário, a casca de arroz, assim como a cinza produzida pela sua queima indiscriminada tornaram-se preocupantes em determinadas regiões do País, com destaque para a região Sul, em virtude do grande volume produzido anualmente.

As pesquisas envolvendo a obtenção de materiais para a construção, empregando a casca de arroz, concentram-se, basicamente, em sua cinza, devido a sua ampla aplicação industrial, com destaque para a indústria cerâmica. Para uso na construção civil (concreto e argamassa), as pesquisas apontam que a cinza deve ser de alta qualidade, com elevado grau de pureza (reduzido teor de carbono) e, principalmente, pela exigência de que apresente elevada reatividade química (estrutura amorfa). Em sentido oposto, citam-se os trabalhos de NAKBANPOTE et al. (2000), DINIZ (2005) e FOLETTO et al. (2005), que apontam os benefícios da cinza que contenha elevado teor de carbono, enfatizando o efeito de suas propriedades adsortivas. Entre as aplicações evidenciadas pelos referidos autores, destaca-se o seu emprego como adsorvente, quer seja na extração de ouro, quer seja na remoção de chumbo e mercúrio em águas residuárias, na remoção de ácidos graxos livres em óleo de soja, na remoção de cobre, níquel e zinco em soluções sintéticas. No caso da agricultura, destaca-se principalmente como substrato (enraizamento; hidroponia em morangueiros; substituto do xaxim no enchimento de vasos para plantas e flores).

No caso da cinza ${ }^{*}$ utilizada na presente pesquisa, ZUCCO \& BERALDO (2005), empregando a metodologia das curvas de evolução da temperatura de hidratação de pastas de cimento com casca passante em peneira de \#200 mesh $(0,074 \mathrm{~mm}$; a massa de casca correspondeu a 7,5\% da massa de cimento), evidenciaram que a adição de cinza foi capaz de minimizar os efeitos negativos dos extrativos presentes na casca de arroz, tendo associado tal comportamento ao elevado teor de carbono. Os referidos autores apontaram que, para a casca na condição natural, parâmetros de avaliação do comportamento químico, como o tempo para atingir a temperatura máxima e a temperatura máxima alcançada, sofreram forte influência da adição de cinza. No caso da casca lavada em solução de cal - LSC, os autores destacam que a influência não foi tão perceptível, tendo constatado a ausência de diferença significativa entre as curvas (parâmetros inclinação máxima, tempo e temperatura máxima) referentes aos percentuais de $20 \%$ e de $35 \%$ de adição de cinza e uma possível influência do aumento de massa no sistema (adições de $20 \%$ e de $35 \%$ de cinza), capaz de reduzir a temperatura máxima alcançada.

Por se tratar de matéria-prima abundante e renovável, tanto a casca como a sua cinza podem ser empregadas como matéria-prima para a obtenção de materiais de construção alternativos, principalmente para atender às necessidades rurais.

Assim, o principal objetivo deste trabalho foi o de avaliar o emprego da cinza da casca de arroz como carga mineral (filler) em misturas cimento-casca. Os efeitos da adição de cinza foram avaliados com base no comportamento mecânico de misturas cimento-cinza-casca de arroz, sendo a mistura cimento-casca considerada como controle (testemunha), tendo sido utilizados corpos-deprova cilíndricos e corpos-de-prova extraídos de placas prensadas como elementos de avaliação do comportamento mecânico.

A principal justificativa para este estudo advém do fato de que o mesmo produtor da casca poderia também ser o da cinza, e por meio da adição de cinza nas misturas cimento-casca estudadas por ZUCCO (1999), seria possível elevar o consumo dos resíduos agroindustriais casca e, principalmente cinza, contribuindo para minimizar os problemas ambientais derivados do descarte inadequado, obtendo-se, então, materiais alternativos para construções rurais.

\footnotetext{
${ }^{*}$ Cinza obtida em processo pirolítico industrial, segundo ROVERE et al. (2002, 2003) e UFRJ (2003).
} 


\section{MATERIAL E MÉTODOS}

A metodologia de trabalho consistiu na caracterização dos materiais em estudo e determinação dos parâmetros de resistência dos produtos resultantes. Ensaios de laboratório foram realizados para a caracterização dos materiais empregados.

\section{Casca de arroz}

Empregaram-se, com base em estudos de ZUCCO (1999), três tipos de distribuição em tamanho de casca: a $10 \mathrm{~mm}$ (casca inteira; módulo de finura $=3,75$ ); a $5 \mathrm{~mm}$ (casca fracionada em moinho-de-martelos, cuja peneira apresentava aberturas com $5 \mathrm{~mm}$ de diâmetro; módulo de finura $=$ $2,91)$, e a $10+5 \mathrm{~mm}$, que é uma mistura das cascas $10 \mathrm{~mm}(65 \%$ em massa $)$ e $5 \mathrm{~mm}(35 \% \mathrm{em}$ massa), apresentando módulo de finura $=3,38$.

\section{Cinza da casca de arroz}

A cinza ensacada (teor de água de $3,1 \%$ e módulo de finura $=3,44$ ) foi cominuída (moinhode-martelos), tendo sido empregada a fração passante em peneira \#100 mesh $(0,149 \mathrm{~mm})$. A análise granulométrica realizada no IME-RJ apontou que cerca de $43 \%$ da cinza possui dimensão superior a 0,074 mm (\# 200). Das micrografias realizadas no IME-RJ, destaca-se a que evidencia a elevada porosidade do material (Figura 1). A composição química da cinza (método: fluorescência de raiosX), realizada pela Central Analítica da UNICAMP, destacou teor de 98\% de carbono.

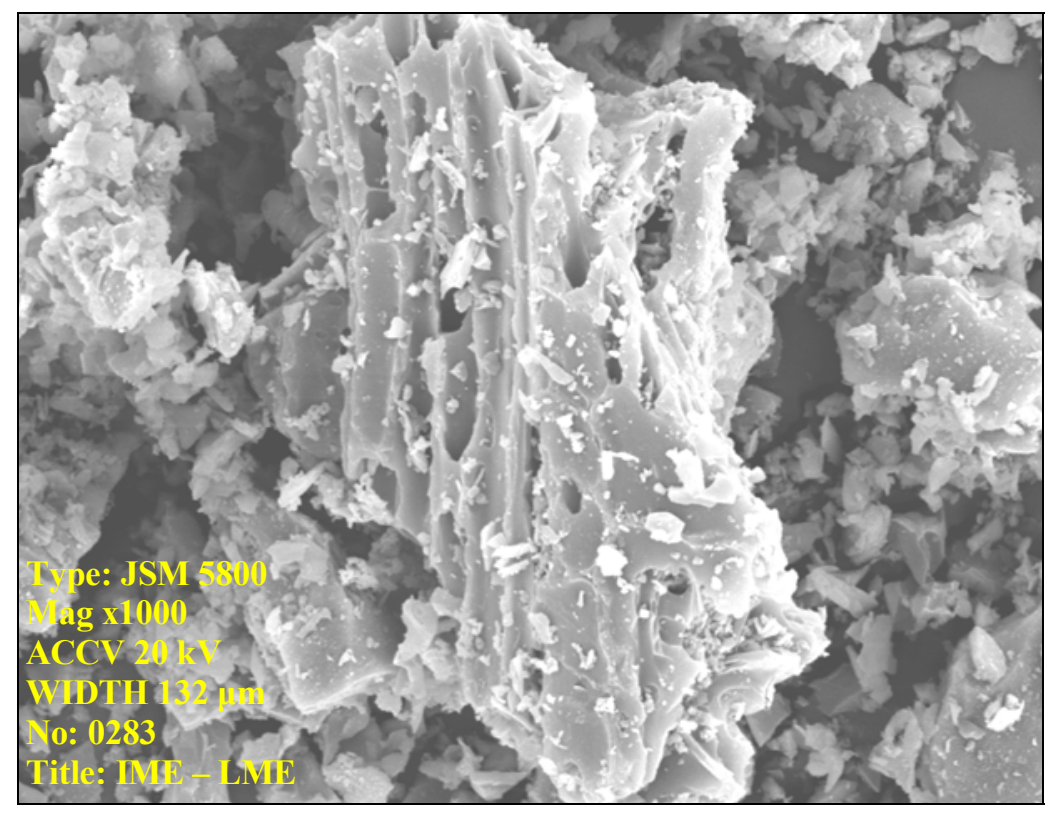

FIGURA 1. Cinza da casca de arroz cominuída (aumento 1.000 X). Rice Husk Ash (RHA) comminuted (magnification 1000 X). Fonte: Izabella Castro Bigno (IME-RJ).

\section{Cimento}

Empregou-se cimento Portland CP-V-ARI RS (NBR 5733 - ABNT, 1991), da marca Votoran, proveniente de um mesmo lote de fabricação, uma vez que sua composição influencia no calor de hidratação.

\section{Acelerador de pega e água de amassamento}

Empregou-se o cloreto de cálcio comercial (3\% em relação à massa de cimento) como acelerador de pega, tendo-se empregado água deionizada como forma de eliminar possíveis fontes de íons (cloretos, fluoretos e carbonatos, entre outros) encontrados na água potável fornecida pela rede pública, capazes de influenciar nos resultados. 


\section{Traços}

Os traços empregados foram baseados em estudos de ZUCCO (1999), tendo-se efetuado a adição de cinza como diferencial. Apresenta-se, na Tabela 1, a descrição dos traços estudados, tendo-se efetuado as misturas com base em unidades de massa.

TABELA 1. Traços (adaptados de ZUCCO, 1999) (cimento:cinza:casca; água/cimento = 0,5). Ratios (adapted from ZUCCO, 1999) (cement: RHA: rice husk; water/cement = 0.5).

\begin{tabular}{cccccc}
\hline Tratamento & Traço & Tratamento & Traço & Tratamento & Traço \\
\hline $10 \mathrm{~mm}-0 \%$ & $1: 0,00: 0,35$ & $5 \mathrm{~mm}-0 \%$ & $1: 0,00: 0,42$ & $10+5 \mathrm{~mm}-0 \%$ & $1: 0,00: 0,39$ \\
$10 \mathrm{~mm}-20 \%$ & $1: 0,20: 0,35$ & $5 \mathrm{~mm}-20 \%$ & $1: 0,20: 0,42$ & $10+5 \mathrm{~mm}-20 \%$ & $1: 0,20: 0,39$ \\
$10 \mathrm{~mm}-35 \%$ & $1: 0,35: 0,35$ & $5 \mathrm{~mm}-35 \%$ & $1: 0,35: 0,42$ & $10+5 \mathrm{~mm}-35 \%$ & $1: 0,35: 0,39$ \\
\hline
\end{tabular}

\section{Obtenção dos corpos-de-prova}

Com base em procedimento descrito por ZUCCO (1999), a mistura mecânica dos materiais foi realizada empregando-se batedeira industrial, tendo-se adotado o seguinte procedimento:

1) Deposição do acelerador de pega no interior da cuba do misturador, com adição da massa total da água deionizada, seguida de mistura até a solubilização do acelerador;

2) Deposição da casca de arroz no interior da cuba, com mistura até a homogeneização (cerca de $30 \mathrm{~s}$ na velocidade 1 - batedeira industrial; $235 \pm 5 \mathrm{rpm}$ );

3) Adição da massa total de cinza (apenas nas misturas com cinza em sua formulação) com mistura até homogeneização (cerca de $30 \mathrm{~s}$ na velocidade 1 - batedeira industrial; $235 \pm 5 \mathrm{rpm}$ ), e

4) Adição da massa total de cimento, homogeneizando-se por 30 s na velocidade 1 , mudandose em seguida para a velocidade 3 da batedeira industrial $(363 \pm 10 \mathrm{rpm})$, de modo que o tempo total de mistura, após a adição do cimento, foi de 3 minutos. Durante o processo de mistura, as paredes internas da cuba foram raspadas de modo a não acumular material.

A mistura foi depositada no interior de um equipamento especificamente desenvolvido para tal fim - a unidade termossônica (UTS), visando à obtenção de cilindros de mistura (Figura 2), ou depositando-se a mistura no interior de uma fôrma metálica (Figura 3) para a obtenção de placas prensadas (pressão de 0,7 MPa). Decorridas $24 \mathrm{~h}$ após a confecção das misturas, efetuava-se a desmoldagem dos cilindros e das placas prensadas, sendo promovida a cura em câmara úmida (ao ar) até a data do ensaio, aos 28 dias de idade, quando então se efetuava o corte dos cilindros/placas prensadas com disco diamantado a seco, para a obtenção de corpos-de-prova utilizados nos diferentes ensaios.

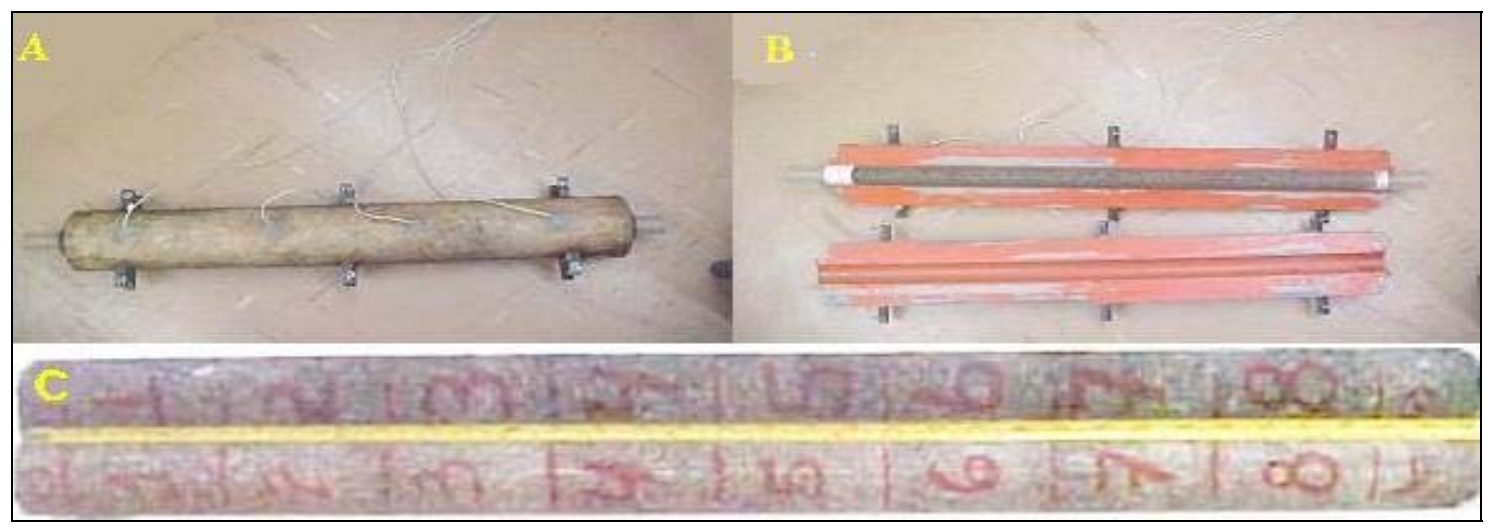

FIGURA 2. Unidade termossônica (A); desmoldagem do cilindro de mistura (B); cilindros de mistura com marcações antes do corte para a obtenção dos corpos-de-prova cilíndricos (C). Unit thermo-sonic (A); de-moulded of the mixture cylinder (B); mixture cylinders with codifications before cutting to obtain the cylindrical specimens $C$ ). 

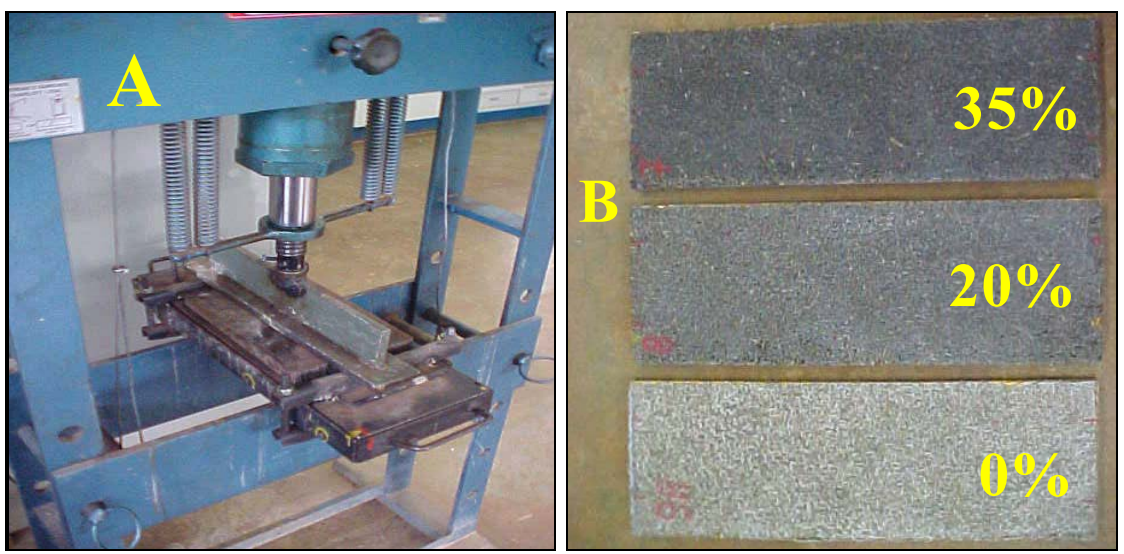

FIGURA 3. Prensa hidráulica convencional e fôrma (A); aspecto das placas prensadas após a desmoldagem, para misturas com adições de $0 \%, 20 \%$ e de $35 \%$ de cinza (B). Conventional hydraulic device and mould (A); aspect of pressed boards after demoulding for mixtures with additions of $0 \%, 20 \%$ and $35 \%$ of RHA (B).

\section{Ensaios realizados com base nas normas NBR 7222 (ABNT, 1994) e NBR 7215 (ABNT, 1996)}

Para avaliar as misturas na forma de corpos-de-prova cilíndricos, o experimento consistiu em um fatorial $3 \times 3$, em que se avaliou o efeito da distribuição em tamanho de casca (Fator CASCA: $5 \mathrm{~mm} ; 10+5 \mathrm{~mm}$ e $10 \mathrm{~mm}$ ) e o efeito da adição de cinza (Fator CINZA: 0\%; 20\% e 35\%), empregados de forma isolada ou em conjunto. De cada cilindro de mistura (cerca de $90 \mathrm{~cm}$ de comprimento e $5 \mathrm{~cm}$ de diâmetro; Figura 4C), foram extraídos corpos-de-prova, utilizando-se de numeração ímpar (1;3; 5 e 7) para o ensaio de tração por compressão diametral (variável: TCD), e o de numeração par (2; 4; 6 e 8) no ensaio por compressão simples (variável: CS), Figuras 4A e 4B.

\section{Ensaios realizados com base na norma ASTM D1037-78 (1982)}

\section{Ensaios de flexão estática}

Neste trabalho de pesquisa, o experimento envolvendo o ensaio de flexão estática (variável Flexão) consistiu em um fatorial $3 \times 3$, por considerar os efeitos atribuídos aos fatores CASCA e CINZA. Assim, aos 28 dias de idade, foi cortado de cada placa (ou repetição) armazenada na câmara úmida (umidade natural, nas condições de laboratório), um corpo-de-prova para ser ensaiado, totalizando três repetições por mistura (Figura 4C).

\section{Ensaios de compressão paralela à superfície}

No ensaio em compressão paralela à superfície (variável CPS), o experimento também consistiu em um fatorial 3 x 3 . Assim, aos 28 dias de idade, foram cortados de cada placa (ou repetição), armazenada na câmara úmida (umidade natural, nas condições de laboratório), oito corpos-de-prova, totalizando 24 repetições por mistura (Figura 4D).

\section{Avaliação do experimento}

As análises estatísticas dos resultados dos ensaios foram efetuadas com o auxílio do software Statgraphics Plus 4.1 (1999), empregando-se o teste de Tukey (comparação entre médias), com nível de significância de 0,05 (95\% de confiança). 

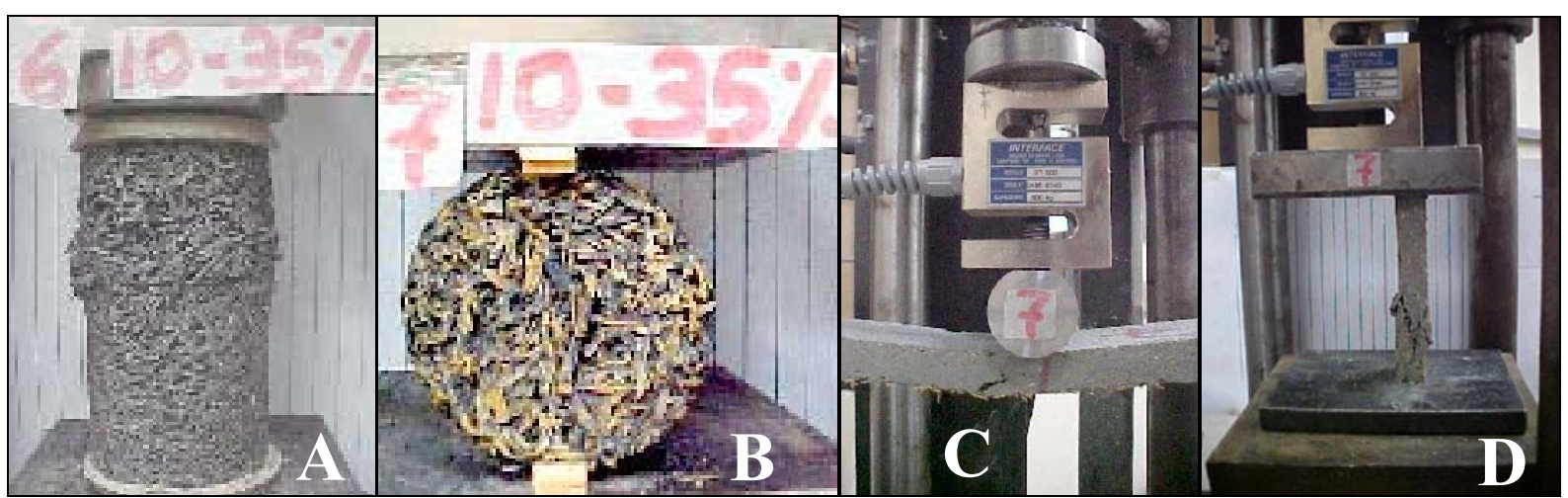

FIGURA 4. Mistura 10 mm-35\%: (A) corpos-de-prova após os ensaios de compressão simples CS; (B) tração por compressão diametral - TCD; (C) flexão estática - Flexão; (D) compressão paralela à superfície - CPS. Mixture 10 mm-35\%: (A) Specimens after simple compression tests - CS, (B) Brazilian Test - TCD, (C) static bending, (D) compression parallel to the surface - CPS.

\section{RESULTADOS E DISCUSSÃO}

Na Tabela 2, apresenta-se o resumo dos resultados dos ensaios mecânicos, para corpos-deprova cilíndricos ensaiados à tração por compressão diametral - TCD, ou à compressão simples CS, e para os corpos-de-prova extraídos das placas prensadas e submetidos à flexão estática Flexão ou à compressão paralela à superfície - CPS.

TABELA 2. Resultado dos ensaios mecânicos (MPa). Results of the mechanical tests (MPa).

\begin{tabular}{|c|c|c|c|c|}
\hline Tratamento & TCD & CS & Flexão & CPS \\
\hline $5 \mathrm{~mm}-0 \%$ & $\begin{array}{c}0,69^{\mathrm{a}, b^{*}} \pm 0,46 \\
(66,68 \%)\end{array}$ & $\begin{array}{c}2,61^{\mathrm{a}} \pm 1,33 \\
(51,05 \%)\end{array}$ & $\begin{array}{c}3,91^{\mathrm{a}} \pm 1,25 \\
(31,87 \%)\end{array}$ & $\begin{array}{c}6,82^{\mathrm{a}, \mathrm{b}, \mathrm{c}} \pm 0,83 \\
(12,21 \%)\end{array}$ \\
\hline $5 \mathrm{~mm}-20 \%$ & $\begin{array}{c}0,49^{\mathrm{a}, \mathrm{b}} \pm 0,11 \\
(22,43 \%)\end{array}$ & $\begin{array}{c}2,74^{\mathrm{a}} \pm 1,14 \\
(41,71 \%)\end{array}$ & $\begin{array}{c}4,00^{\mathrm{a}} \pm 1,15 \\
(28,83 \%)\end{array}$ & $\begin{array}{c}6,56^{\mathrm{a}, \mathrm{b}} \pm 1,18 \\
(17,91 \%)\end{array}$ \\
\hline $5 \mathrm{~mm}-35 \%$ & $\begin{array}{c}1,02^{\mathrm{a}, \mathrm{b}} \pm 0,32 \\
(31,71 \%)\end{array}$ & $\begin{array}{c}3,08^{\mathrm{a}} \pm 0,46 \\
(15,04 \%)\end{array}$ & $\begin{array}{c}4,00^{\mathrm{a}} \pm 0,68 \\
(17,01 \%)\end{array}$ & $\begin{array}{c}7,52^{b, c, d} \pm 1,62 \\
(21,59 \%)\end{array}$ \\
\hline $10+5 \mathrm{~mm}-0 \%$ & $\begin{array}{c}1,10^{b} \pm 0,44 \\
(40,34 \%)\end{array}$ & $\begin{array}{c}3,35^{\mathrm{a}} \pm 2,38 \\
(71,16 \%)\end{array}$ & $\begin{array}{c}4,92^{\mathrm{a}} \pm 0,58 \\
(11,72 \%)\end{array}$ & $\begin{array}{c}8,56^{\mathrm{e}} \pm 0,79 \\
(9,25 \%)\end{array}$ \\
\hline $10+5 \mathrm{~mm}-20 \%$ & $\begin{array}{c}1,00^{a, b} \pm 0,21 \\
(20,51 \%)\end{array}$ & $\begin{array}{c}2,33^{\mathrm{a}} \pm 0,60 \\
(25,89 \%)\end{array}$ & $\begin{array}{c}3,68^{\mathrm{a}} \pm 0,68 \\
(18,33 \%)\end{array}$ & $\begin{array}{c}7,49^{\mathrm{b}, \mathrm{c}, \mathrm{d}} \pm 0,97 \\
(12,95 \%)\end{array}$ \\
\hline $10+5 \mathrm{~mm}-35 \%$ & $\begin{array}{c}0,34^{\mathrm{a}} \pm 0,15 \\
(43,63 \%)\end{array}$ & $\begin{array}{c}2,21^{\mathrm{a}} \pm 1,14 \\
(51,61 \%)\end{array}$ & $\begin{array}{c}4,21^{\mathrm{a}} \pm 0,91 \\
(21,55 \%)\end{array}$ & $\begin{array}{c}7,41^{\mathrm{a}, \mathrm{b}, \mathrm{c}, \mathrm{d}} \pm 1,11 \\
(15,01 \%)\end{array}$ \\
\hline $10 \mathrm{~mm}-0 \%$ & $\begin{array}{c}0,98^{\mathrm{a}, \mathrm{b}} \pm 0,33 \\
(33,15 \%)\end{array}$ & $\begin{array}{c}3,08^{\mathrm{a}} \pm 0,47 \\
(15,11 \%)\end{array}$ & $\begin{array}{c}5,09^{\mathrm{a}} \pm 0,58 \\
(11,33 \%)\end{array}$ & $\begin{array}{c}8,23^{\mathrm{d}, \mathrm{e}} \pm 0,94 \\
(11,38 \%)\end{array}$ \\
\hline $10 \mathrm{~mm}-20 \%$ & $\begin{array}{c}0,57^{\mathrm{a}, \mathrm{b}} \pm 0,13 \\
(23,58 \%)\end{array}$ & $\begin{array}{c}1,79^{\mathrm{a}} \pm 0,58 \\
(32,51 \%)\end{array}$ & $\begin{array}{c}3,97^{\mathrm{a}} \pm 1,07 \\
(26,88 \%)\end{array}$ & $\begin{array}{c}7,72^{\mathrm{c}, \mathrm{d}, \mathrm{e}} \pm 1,03 \\
(13,30 \%)\end{array}$ \\
\hline $10 \mathrm{~mm}-35 \%$ & $\begin{array}{c}1,11^{b} \pm 0,43 \\
(39,05 \%)\end{array}$ & $\begin{array}{c}3,34^{\mathrm{a}} \pm 2,38 \\
(71,20 \%)\end{array}$ & $\begin{array}{c}4,18^{\mathrm{a}} \pm 0,66 \\
(15,86 \%)\end{array}$ & $\begin{array}{c}6,49^{\mathrm{a}} \pm 0,95 \\
(14,57 \%)\end{array}$ \\
\hline
\end{tabular}

* Valores médios \pm desvio-padrão (coeficiente de variação). Em cada coluna, médias seguidas de mesma letra não diferem entre si, pelo teste de Tukey, a 5\% de probabilidade.

Avaliando-se a resistência mecânica das misturas por meio de corpos-de-prova cilíndricos, verificou-se que apenas o ensaio de tração por compressão diametral (TCD) evidenciou a existência de diferença estatística significativa, mas apenas entre a mistura $10+5 \mathrm{~mm}-35 \%$ e as misturas $10 \mathrm{~mm}-35 \%$ e $10+5 \mathrm{~mm}-0 \%$. Os elevados coeficientes de variação obtidos, e também para o caso da variável CS, indicam que esses tipos de ensaio provavelmente não sejam os mais indicados para avaliar o comportamento de tais misturas binárias (casca e cinza). 
No caso da resistência mecânica dos corpos-de-prova extraídos das placas prensadas, verificou-se que apenas no ensaio de compressão paralela à superfície (CPS) foi possível evidenciar a existência de diferença estatística significativa, o que se atribuiu ao efeito de adição da cinza, que promoveu aumento no percentual de finos nas misturas. Esse fato pode ser explicado, em parte, com base nas observações de RODRIGUES \& GHAVAMI (2004), para os quais valores elevados de cinza afetam negativamente a adesão entre partículas. Também se observa a ocorrência de coeficientes de variação muito inferiores aos dos casos precedentes, denotando a maior uniformidade no processo de fabricação das placas prensadas.

Avaliando-se o comportamento geral das misturas, verifica-se variabilidade anormal nos resultados de resistência mecânica, podendo ser associado tal fato às interações químicas da cinza com o aglomerante, conforme fora evidenciado por ZUCCO \& BERALDO (2005).

Com base nessas considerações, fica implícita a necessidade de se definir o teor mínimo de cinza para cada mistura, que seja capaz de neutralizar a ação negativa dos extrativos liberados para a água de amassamento, com base na capacidade adsortiva da cinza, pois a mesma influencia no comportamento químico da pasta de cimento.

Contudo, essa afirmação depende de metodologia de avaliação apropriada (ainda não definida), que correlacione teores de extrativos com a capacidade adsortiva da cinza (ambos dependentes de análises específicas), tendo em vista o fato de que os "agregados" CASCA e CINZA apresentam características reativas, interferindo no processo de hidratação da pasta de cimento.

Destaca-se, no caso de concretos e argamassas, que os efeitos dos agregados reativos (reação álcali-agregado) já são bem conhecidos. No caso dos compósitos, os estudos efetuados por ZUCCO (1999) apontam que os extrativos presentes nos "agregados" determinam o comportamento inicial de suas misturas com o cimento, avaliado indiretamente por meio de parâmetros como o tempo necessário para o início e o fim de pega, o tempo para atingir a temperatura máxima, a evolução da resistência à penetração, dentre outros ${ }^{\dagger}$.

A extensão dessa influência não pôde ser avaliada, de modo que se torna prudente recomendar o desenvolvimento de estudos em relação às características químicas dos materiais empregados. Destacam-se, nesse caso, as observações de ZUCCO (1999), em que os métodos por ele avaliados demonstraram ser incapazes de prever particularidades das diferentes fitomassas empregadas, conduzindo, geralmente, a resultados totalmente antagônicos, associados aos efeitos sinérgicos ou antagônicos dos extrativos presentes na fitomassa sobre as reações químicas do cimento, como também aponta a literatura especializada.

Verificou-se, ainda, que o percentual de adição de $0 \%$ de cinza (controle ou testemunha) apresentou, em geral, os valores mais elevados de resistência média, porém não diferindo significativamente dos demais percentuais de adição. Destaca-se que o coeficiente de correlação entre a resistência à tração por compressão diametral (TCD) e a resistência à compressão simples (CS) não foi elevado $(\mathrm{R}=0,62)$.

Avaliando-se os valores médios de resistência à TCD e à Flexão, destacou-se tendência de redução no valor das resistências com o aumento no teor de casca de arroz, ou seja, a distribuição em tamanho de casca, definida como $10 \mathrm{~mm}$, entra na composição das misturas com percentual de adição de $35 \%$ em relação à massa de cimento, enquanto a distribuição em tamanho de casca, definida como $5 \mathrm{~mm}$, entra na composição das misturas com percentual maior de adição, de $42 \%$ em relação à massa de cimento.

No caso dos ensaios de CS e de CPS, as resistências médias não permitem evidenciar comportamento que possibilite emitir considerações mais aprofundadas envolvendo a quantidade de

\footnotetext{
${ }^{\dagger}$ Ensaios e avaliações discutidos em capítulo específico, constantes na Tese de Doutorado do primeiro autor.
} 
casca ou a dimensão de suas partículas, pois outros fatores, como o efeito da prensagem aplicado às placas, interferem na análise.

Com base nos resultados de resistência apresentados na Tabela 2, procurou-se verificar a existência de correlação entre os resultados dos diferentes ensaios. As equações lineares e seus respectivos coeficientes de correlação entre o ensaio de flexão estática (Flexão) e os ensaios de tração por compressão diametral (TCD), compressão simples (CS) e o de compressão paralela à superfície (CPS) são apresentados na Tabela 3.

TABELA 3. Modelos matemáticos. Mathematical models.

\begin{tabular}{lc}
\hline \multicolumn{1}{c}{ Equação Linear } & $\mathrm{R}$ \\
\hline Flexão $=3,15261+1,54071$ TCD & 0,52 \\
Flexão $=2,96216+0,553335$ CS & 0,65 \\
Flexão $=-0,527226+0,639343$ CPS & 0,69 \\
\hline
\end{tabular}

VALENZUELA (1989) verificou que, para a mesma mistura madeira-cimento, a correlação entre os resultados dos ensaios de flexão estática (placas) e os de tração por compressão diametral de corpos-de-prova cilíndricos foi de 0,76 , enquanto a correlação entre a flexão estática das placas e a compressão simples de corpos-de-prova cilíndricos foi de 0,63 . $\mathrm{O}$ autor fez, ainda, referência ao fato de que, na bibliografia especializada, não se encontravam referências quanto a essa relação entre placas e corpos-de-prova cilíndricos.

A ausência de maiores informações envolvendo a correlação entre os resultados dos ensaios de corpos-de-prova cilíndricos e os de placas prensadas evidenciou a necessidade de se desenvolverem estudos mais aprofundados envolvendo o processo de obtenção de corpos-de-prova cilíndricos e o das placas prensadas, pois a casca de arroz apresenta particularidades específicas, associadas a sua natureza diferenciada de forma e de composição química. O processo de prensagem adotado deve ser similar àquele aplicado às placas.

Em relação às misturas avaliadas, destaca-se que a combinação da casca de arroz $10 \mathrm{~mm}$ com a adição de $35 \%$ de cinza da casca de arroz apresenta as maiores vantagens na produção do compósito, pois se obtém redução de cerca de $25 \%$ no consumo de cimento (comparando-se com a mistura $10 \mathrm{~mm}-0 \%$ ) sem afetar de forma significativa a resistência mecânica das placas prensadas.

Do ponto de vista ambiental, a capacidade de confinamento ou de não-emissão de carbono para a atmosfera ${ }^{\ddagger}$, representada pela mistura $10 \mathrm{~mm}-35 \%$ (traço 1:0,35:0,35), com base na composição teórica da casca de arroz (51\% de carbono) e da cinza ( $98 \%$ de carbono), foi a melhor, pois cada tonelada de cimento consumido no processo de fabricação das placas reterá em sua pasta cerca de 1,9 tonelada de $\mathrm{CO}_{2}$, contribuindo, dessa forma, para a redução na emissão de $\mathrm{CO}_{2}$, podendo tornar as construções rurais mais viáveis do ponto de vista ecológico.

\section{CONCLUSÕES}

O efeito químico (negativo) da casca de arroz sobre a pasta de cimento foi minimizado com a adição de cinza, empregada como filler. Esse efeito não pôde ser quantificado de forma adequada, uma vez que o efeito combinado cinza-casca se traduziu, em boa parte das análises, na ausência de diferença estatística significativa nos ensaios de resistência à tração por compressão diametral, à compressão simples e em flexão estática.

As análises apresentadas, restritas ao contexto, evidenciam a necessidade de obtenção dos corpos-de-prova, empregando-se os mesmos critérios de fabricação, uma vez que os valores de correlação encontrados são considerados pouco adequados para a validação de equações preditivas de comportamento das placas.

\footnotetext{
* Método de cálculo: quantidade de casca ou de cinza x teor de carbono x 3,67 (relação $\mathrm{CO}_{2} / \mathrm{C}$ ) = quantidade equivalente de $\mathrm{CO}_{2}$ constituinte. A soma das quantidades dos constituintes casca e cinza perfazem o total retido na mistura.
} 
Ressalta-se que a mistura $10 \mathrm{~mm}-35 \%$ apresentou os melhores valores médios de resistência nos ensaios mecânicos de corpos-de-prova cilíndricos, contudo não diferindo significativamente das demais misturas. Destaca-se que, em comparação com a mistura 10 mm-0\%, a mistura 10 mm-35\% diferiu significativamente apenas no ensaio de resistência à compressão paralela à superfície.

A ausência de parâmetros mais adequados para avaliar as interações físico-químicomecânicas evidencia a necessidade de serem desenvolvidas novas pesquisas sobre esse assunto. Contudo, a possibilidade de utilização de tais resíduos vegetais mostra-se favorável, pois quanto maior for o consumo da casca de arroz e de suas cinzas, para a mesma faixa de resistência, maior será o ganho ambiental.

\section{AGRADECIMENTOS}

Aos colaboradores, URBANO Agroindustrial (www.urbano.com.br); Izabella C. Bigno (izabella@ime.eb.br); Eliezer Laister (eliezerl@votoran.com.br); Sidney Ohta (interface@mpcnet.com.br) células de carga, caixas de leitura e de aquisição de dados; Marcelo (www.jsl.com.br) unidades hidráulicas; Leandro Morais (morais@agr.unicamp.br) sistemas de aquisição de dados; Luiz C. Silva (luiz.silva@agr.unicamp.br) protótipos; José Benedito dos Santos (jose.santos@agr.unicamp.br) fôrmas em fibra, e Flávia F. Vieira (flavia@agr.unicamp.br) ensaios, que possibilitaram a realização desta pesquisa.

\section{REFERÊNCIAS}

ASTM. AMERICAN SOCIETY FOR TESTING AND MATERIAL. ASTM D1037-78. Standard methods of evaluating the properties of wood-based fiber and particle panel materials. In: Annual Book of ASTM Standards, Philadelphia, 1982.

ABNT. ASSOCIAÇÃO BRASILEIRA DE NORMAS TÉCNICAS. NBR 5733: Cimento Portland de alta resistência inicial, especificação. Rio de Janeiro, 1991. 5 p.

. NBR 7215: Cimento Portland: determinação da resistência à compressão. Método de ensaio. Rio de Janeiro, 1996. 8 p.

. NBR 7222: Argamassa e concreto: determinação da resistência à tração por compressão diametral de corpos-de-prova cilíndricos. Método de ensaio. Rio de Janeiro, 1994. 3 p.

DINIZ, J. Conversão térmica de casca de arroz à baixa temperatura: produção de bioóleo e resíduo sílico-carbonoso adsorvente. 2005. 185 f. Tese (Doutorado em Química) - Universidade Federal de Santa Maria, Santa Maria, 2005.

FOLETTO, E.L.; HOFFMANN, R.; HOFFMANN, R.S.; PORTUGAL JÚNIOR, U.L.; JAHN, S.L. Aplicabilidade das cinzas da casca de arroz. Química Nova, São Paulo, v.28, n.6, p.1.055-60, 2005.

NAKBANPOTE, W.; THIRAVETYAN, P.; KALAMBAHETI, C. Preconcentration of gold by rice husk ash. Minerals Engineering, Oxford, v.13, n.4, p.391-400, 2000.

RODRIGUES, C. de S.; GHAVAMI, K. Efeitos da adição de cinza de casca de arroz no comportamento mecânico de compósitos cimentícios reforçados por polpas de bambu. In: BRASILNOCMAT 2004: CONFERÊNCIA BRASILEIRA DE MATERIAIS E TECNOLOGIAS NÃOCONVENCIONAIS: HABITAÇÕES E INFRA-ESTRUTURA DE INTERESSE SOCIAL, 2004, Pirassununga. Anais... . Pirassununga: FZEA/USP, 2004. p. 440-50. 1 CD-ROM.

ROVERE, E.L. La (Coord.); D’AVIGNON, A.; MUYLAERT, M.S.; PIMENTEIRA, C.; OLIVEIRA, L.B; ROSA, L.P. Estudo de viabilidade de projeto voltado à adoção de mecanismo de desenvolvimento limpo (MDL): A utilização da casca de arroz na co-geração de energia e a decorrente mitigação de gases que contribuem para o efeito estufa como mecanismo de desenvolvimento limpo. Rio de Janeiro: IVIG/LIMA/COPPE/UFRJ, 2002. 24 p. Disponível em: http://www.centroclima.org.br/ccpdf/. Acesso em: 27 nov. 2003. Relatório preliminar 
ROVERE, E.L. La (Coord.); D’AVIGNON, A.; MUYLAERT, M.S.; PIMENTEIRA, C.; HENRIQUES, R.M. Estudo de viabilidade de projeto voltado à adoção de mecanismo de desenvolvimento limpo (MDL): A utilização da casca de arroz na co-geração de energia e a decorrente mitigação de gases que contribuem para o efeito estufa como mecanismo de desenvolvimento limpo. Síntese. Rio de Janeiro, agosto de 2003. 13 p. Disponível em: http://www.centroclima.org.br/ccpdf/. Acesso em: 27 dez. 2007.

STATGRAPHICS Plus Version 4.1. User Manual. Rockville: Manugistics, 1999.

UNIVERSIDADE FEDERAL DO RIO DE JANEIRO. Estudo de viabilidade de projeto voltado à adoção de mecanismo de desenvolvimento limpo (MDL): A utilização da casca de arroz na cogeração de energia e a decorrente mitigação de gases que contribuem para o efeito estufa como mecanismo de desenvolvimento limpo. Rio de Janeiro: Fundação COPPETEC. Relatório Final. 109 p. Disponível em: http://www.centroclima.org.br/ccpdf/. Acesso em: 28 dez. 2007.

VALENZUELA, W. Contribution à la determination de l'aptitude d'essences forestières pour la fabrication de panneaux de fibro-ciment. 1989. 229 f. Thèse (Docteur en Sciences Agronomiques) Faculté des Sciences Agronomiques, Gembloux, 1989.

ZUCCO, L.L. Estudo da viabilidade de fabricação de placas de compósitos à base de cimento e casca de arroz. 1999. 118 f. Dissertação (Mestrado em Construções Rurais e Ambiência) Faculdade de Engenharia Agrícola, Universidade Estadual de Campinas, Campinas, 1999.

ZUCCO, L.L.; BERALDO, A.L. Influência da casca de arroz e de sua cinza sobre a hidratação da pasta de cimento de alta resistência inicial (CP-V-ARI). In: IAC-NOCMAT 2005: CONFERÊNCIA INTERAMERICANA SOBRE MATERIAIS E TECNOLOGIAS NÃO-CONVENCIONAIS NA CONSTRUÇÃO ECOLÓGICA E SUSTENTÁVEL, 2005, Rio de Janeiro. Anais... Rio de Janeiro: ABMTENC, 2005. 1 CD-ROM. 